\title{
Capsule Commentary on Lin et. al. Empirical Comparison of Publication Bias Tests in Meta-analysis
}

\author{
Akira Kuriyama, $\mathrm{MD}, \mathrm{MPH}^{1,2}$ \\ 'Department of General Internal Medicine, Kurashiki Central Hospital, Okayama, Japan; ${ }^{2}$ Emergency and Critical Care Center, Kurashiki Central \\ Hospital, Okayama, Japan.
}

J Gen Intern Med 33(8):1382

DOI: $10.1007 / \mathrm{s} 11606-018-4528-1$

(c) Society of General Internal Medicine 2018

tudies with statistically significant findings are more likely to be published than those with non-significant findings. This publication bias is a threat to consumers of metaanalyses, because it can exaggerate the evidence supporting treatment and hence mislead decision-making. Some statistical tests have been developed in an attempt to detect and adjust for this bias.

In this study, Lin et al. assessed for the presence of publication bias in 28,655 trials using 7 publication bias tests. ${ }^{1}$ Consistent with previous findings, they found that Egger's regression test more frequently detected publication bias than other tests. ${ }^{2}$ The authors also found that, while there was strong agreement of the results among Tang's, Macaskill's, Deeks', and Peters' regression tests for binary outcomes, the agreement among the remaining comparisons for publication bias tests was only weak or moderate. This suggests that metaanalysts cannot completely rely on a single publication bias test, and reminds researchers and consumers of meta-analyses to carefully interpret the results even when there is the absence of statistical evidence of publication bias.

What can we do given that no test perfectly assures publication bias? There is no single, magical solution. First, systematic reviewers should resort to non-statistical approaches, as the authors suggest. These represent an exhaustive search for gray literature and a contact for unpublished details through clinical trial registries and drug approval agencies. Second, researchers of original studies need to register trials and primary outcomes on clinical trial registries, and to report their pre-specified outcomes whether or not they are significant. $^{3}$ This will prevent non-significant results from being buried. Third, reviewers and editors need to endorse the reporting guidelines (ex, CONSORT) for original studies. ${ }^{4}$ This allows confirmation of or request for the otherwise unpublished results by looking through study protocols or trial registries. Finally, consumers of meta-analyses need to remain cautious because it is impossible to identify all unpublished data. As Albert Einstein once said, "Unthinking respect for authority is the greatest enemy of truth."

Corresponding Author: Akira Kuriyama, MD, MPH; Department of General Internal Medicine, Kurashiki Central Hospital, Okayama, Japan (e-mail: akira.kuriyama.jpn@gmail.com).

\section{Compliance with Ethical Standards:}

Conflict of Interest: The author declares that he does not have a conflict of interest.

\section{REFERENCES}

1. Lin L, Chu H, Hassan M, Hong C, Qu Z, Cole SR, Chen Y. Empirical comparison of publication bias tests in meta-analysis. J Gen Intern Med https://doi.org/10.1007/s11606-018-4425-7

2. Peters JL, Sutton AJ, Jones DR, Abrams KR, Rushton L. Comparison of two methods to detect publication bias in meta-analysis. JAMA 2006; 295(6):676-80.

3. Schulz KF, Altman DG, Moher D; CONSORT Group. CONSORT 2010 statement: updated guidelines for reporting parallel group randomised trials. BMJ. 2010; 340:c332.

4. Kuriyama A, Takahashi N, Nakayama T. Reporting of critical care trial abstracts: a comparison before and after the announcement of CONSORT guideline for abstracts. Trials 2017; 18(1):32. 